\title{
Diagnóstico nutricional das crianças atendidas no projeto de extensão AMAR - Assistência Multidisciplinar em Pediatria
}

\author{
Nutritional diagnosis of children assisted in the AMAR extension project
}

Fabiana Migliaccio Mansur ${ }^{1}$, Isabel Rey Madeira ${ }^{2}$, Lara Pimenta Narciso ${ }^{3}$, Ana Paula Córdova da Costa ${ }^{4}$, Antônia da Conceição Cylindro Machado ${ }^{5}$, Simone Augusta Ribas ${ }^{6}$, Celise Regina Alves da Motta Meneses ${ }^{7}$, Vinicius Anciães Darriba ${ }^{8}$, Dayse Silva de Carvalho ${ }^{9}$

\author{
1 Aluna de graduação. Faculdade de Ciências Médicas da Universidade do Estado do Rio de Janeiro (FCM-UERJ), Brasil. \\ E-mail: fabianammansur92@gmail.com \\ 2 Coordenadora do Projeto Amar e Adjunta do Departamento de Pediatria. Faculdade de Ciências Médicas da Universidade do \\ Estado do Rio de Janeiro (FCM-UERJ), Brasil. E-mail: isamadeira@oi.com.br \\ 3 Aluna de graduação. Faculdade de Ciências Médicas da Universidade do Estado do Rio de Janeiro (FCM-UERJ), Brasil. \\ E-mail: laranarciso@hotmail.com \\ 4 Fonoaudióloga. Hospital Universitário Pedro Ernesto da Universidade do Estado do Rio de Janeiro (HUPE-UERJ), Brasil. \\ E-mail: apcordova@gmail.com \\ 5 Enfermeira. Hospital Universitário Pedro Ernesto da Universidade do Estado do Rio de Janeiro (HUPE-UERJ), Brasil. \\ E-mail: haccmachado@oi.com.br \\ 6 Nutricionista. Hospital Universitário Pedro Ernesto da Universidade do Estado do Rio de Janeiro (HUPE-UERJ), Brasil. \\ E-mail: ribasnut@yahoo.com.br \\ 7 Médica. Unidade Docente Assistencial de Pediatria do Hospital Universitário Pedro Ernesto da Universidade do Estado do Rio de \\ Janeiro (HUPE-UERJ), Brasil. E-mail: celisemeneses@gmail.com \\ 8 Professor Adjunto. Instituto de Psicologia da Universidade do Estado do Rio de Janeiro (UERJ), Brasil. \\ E-mail: vdarriba@centroin.com.br \\ 9 Assistente social. Hospital Universitário Pedro Ernesto da Universidade do Estado do Rio de Janeiro (HUPE-UERJ), Brasil. \\ E-mail: sersocialchefia.hupe@gmail.com
}

Recebido em: 18/05/2016 | Aprovado em: 31/01/2017

DOI: $10.12957 /$ interag.2017.22864

\section{Resumo}

O Projeto AMAR - Assistência Multidisciplinar em Pediatria - é um projeto de extensão universitária do Departamento de Pediatria da Faculdade de Ciências Médicas da Universidade do Estado do Rio de Janeiro (UERJ), de cunho multidisciplinar, implantado no Ambulatório de Pediatria do Hospital Universitário Pedro Ernesto da UERJ (HUPE-UERJ). O AMAR busca aprimorar a assistência, o ensino e a pesquisa em atenção primária à criança. Neste projeto de pesquisa objetivou-se realizar o diagnóstico clínico nutricional das crianças atendidas no Projeto AMAR ao longo dos cinco primeiros anos de vida. Com esta finalidade, foi realizado um estudo de observação longitudinal nas crianças nascidas no Núcleo Perinatal do HUPE-UERJ que foram incluídas e que estavam em acompanhamento no Projeto AMAR. Foi realizado monitorização crescimento e

\begin{abstract}
The Project AMAR, in English PMA (Pediatric Multidisciplinary Assistance), is an extension project of the Department of Pediatrics, of the School of Medical Sciences of the Rio de Janeiro State University (UERJ). Of a multidisciplinary nature, the project has been established in the Pedro Ernesto University Hospital of UERJ, also known as HUPE-UERJ. AMAR seeks to improve care, education and research focused primarily on children. This research project aimed at making the clinical nutritional diagnosis of children treated in the AMAR project over the course of their first five years of life. With this purpose, a longitudinal observational study was carried out on children that were born in the Perinatal Center of HUPE-UERJ and who were included in the project's follow up. Monitoring of growth and nutritional development was assessed based on a multidisciplinary protocol. The results were obtained by recording dietary information
\end{abstract}


do desenvolvimento baseado em um protocolo multidisciplinar, contendo recordatório alimentar aplicado em cada consulta, com ênfase em dados sobre o aleitamento materno e alimentação complementar no primeiro ano de vida. A avaliação e a classificação nutricional foram realizadas pela antropometria de acordo com o proposto pelo Ministério da Saúde e pela Organização Mundial de Saúde. A compilação dos dados obtidos revelou maioria eutrófica dentre as crianças acompanhadas até o quinto ano de vida, com frequência de obesidade, sobrepeso e magreza inferiores aos índices nacionais. Foram encontrados índices superiores à média em outros estados da região sudeste para aleitamento materno em qualquer tempo, o que torna efetiva a abordagem multidisciplinar realizada no Projeto AMAR.

Palavras-chave: Pediatria; Aleitamento Materno; Obesidade.

Área temática: Saúde.

Linha de extensão: Infância e Adolescência. at every appointment, with emphasis on the data regarding breastfeeding and complementary feeding during the first year of life. Nutritional evaluation was made through anthropometry and carried out following the classification proposed by the Ministry of Health and by the World Health Organization, classifying them according to their nutritional status. The compilation of the obtained data revealed mostly eutrophic children among those accompanied until the fifth year of life. Frequently, obesity, overweight and thinness were below the national rates. Higher levels of breastfeeding were reported, compared to the other states of the southeast region, at any time, which makes the multidisciplinary approach adopted in the Project AMAR effective.

\section{Introdução}

A nutrição adequada é fundamental para o crescimento e o desenvolvimento saudável das crianças. O aleitamento materno exclusivo é a forma de alimentação indicada para o lactente nos seis primeiros meses de vida. ${ }^{1}$ Após esse período, se faz necessária a introdução gradual de alimentação complementar a fim de atender às necessidades nutricionais nessa nova fase da vida, respeitando a maturidade fisiológica e a individualidade da criança. ${ }^{2}$ Para esta prática, não bastam normas rígidas ou regras e orientações ditadas. Sua construção, a partir do conhecimento científico e do embasamento teórico, tem como agente central a família, fundamental na busca das alternativas e das soluções para as questões específicas da alimentação da criança.

O projeto AMAR - Assistência Multidisciplinar em Pediatria - assiste crianças desde a alta do Núcleo Perinatal do Hospital Universitário Pedro Ernesto da Universidade do Estado do Rio de Janeiro (HUPE-UERJ), inserido na rede de hospitais da Iniciativa Hospital Amigo da Criança ${ }^{3}$, até cinco anos de idade, visando à promoção 
de saúde e à prevenção de seus agravos, através de atendimento de puericultura multiprofissional no Ambulatório de Pediatria do mesmo hospital. Também serve de base para o ensino e a pesquisa, através do treinamento de alunos de graduação e pósgraduação de diversas áreas de saúde - enfermagem, fonoaudiologia, medicina, nutrição, psicologia e serviço social. A pesquisa inserida no projeto tem como foco a avaliação do impacto da puericultura multidisciplinar na saúde das crianças e na qualidade da assistência $^{4}$

Uma das maiores demandas de ensino, assistência e pesquisa no Projeto AMAR é a alimentação infantil. Visando à promoção do crescimento e do desenvolvimento saudáveis de sua clientela, os profissionais de saúde, professores e seus alunos envolvidos no projeto, entendendo que a questão nutricional merece uma visão multidisciplinar e integral, vêm aprimorando não só a assistência, mas também o ensino, no tocante à nutrição infantil. A troca de saberes entre as diversas categorias envolvidas no Projeto AMAR, e destas com sua clientela, tem proporcionado uma melhor adesão da família à prescrição nutricional, e ao crescimento e desenvolvimento adequados das crianças.

A obesidade na infância vem emergindo como um problema de saúde pública em todo o mundo, inclusive no Brasil, e na faixa etária pediátrica. ${ }^{5}$ Sabe-se que esta doença tem papel central na fisiopatologia da síndrome metabólica na infância. Esta síndrome agrega os fatores de risco para doença cardiovascular, quer sejam, além da obesidade, a dislipidemia, a hipertensão e a glicemia alterada, e já pode ser aplicada à criança. Sua prevenção se inicia no princípio da vida, com estilo de vida saudável, tendo a nutrição saudável papel predominante. ${ }^{6}$

Neste contexto, o objetivo da presente pesquisa é realizar diagnóstico clínico nutricional das crianças atendidas no projeto AMAR do nascimento até o quinto ano de vida, e caracterizar o aleitamento materno e a introdução dos alimentos complementares no primeiro ano de vida. Para tal, o grupo foi estudado quanto à alimentação ao longo do primeiro ano de vida, com ênfase nos períodos de aleitamento materno exclusivo e 
aleitamento materno preferencial, no término da amamentação e na introdução dos alimentos complementares.

\section{Métodos}

Foi realizado um estudo longitudinal nas crianças acompanhadas pelo Projeto AMAR que completaram cinco anos de idade. As crianças foram estudadas a partir dos dados compilados nos prontuários, desde a primeira consulta - realizada em até 15 dias de vida - até cinco anos de idade completos. Ao total, 77, 50, 38, 26 e 14 crianças que completaram respectivamente $1,2,3,4$ e 5 anos de idade foram estudadas.

Foi empregado um protocolo multidisciplinar de atendimento centrado na monitorização do crescimento e do desenvolvimento, alimentação e prevenção e diagnóstico das principais doenças da infância. A caracterização da alimentação ao longo do primeiro ano de vida das crianças estudadas foi feita através de recordatório alimentar colhido a cada consulta no ambulatório, com ênfase no período de aleitamento materno exclusivo, aleitamento materno preferencial, término do aleitamento materno e introdução dos alimentos complementares.

Definiu-se como aleitamento materno exclusivo aquele no qual a criança recebe somente leite materno, seja direto da mama ou ordenhado, ou leite humano de outra fonte, sem outros líquidos ou sólidos, com exceção de gotas ou xaropes contendo vitaminas, sais de reidratação oral, suplementos minerais ou medicamentos. Por sua vez, aleitamento materno preferencial é observado quando a criança recebe, além do leite materno, água ou bebidas à base de água, sucos de frutas e fluidos rituais. ${ }^{2}$

Para avaliação nutricional, realizou-se a antropometria aplicada à classificação proposta pelo Ministério da Saúde, que utiliza como referenciais de peso, comprimento e índice de massa corpórea (IMC) aqueles da Organização Mundial de Saúde (OMS). ${ }^{7,8}$ De acordo com esta classificação, as crianças de até 5 anos de idade são classificadas com obesidade, sobrepeso, risco de sobrepeso, eutrofia, magreza e magreza acentuada, conforme o intervalo entre percentis nos quais se encaixam. Desse modo, foram 
classificadas com obesidade aquelas crianças cujo IMC encontrava-se acima do escore $z+3$; com sobrepeso aqueles com IMC maior que escore $z+2$; com risco de sobrepeso os com IMC maior que o escore $z+1$; eutróficos aqueles com IMC entre escore $z-2$ e +1 ; magreza quando IMC está < escore z -2; e, por último, magreza acentuada quando o IMC for menor que $<$ escore $z-3.1$

Valores do índice altura para idade inferiores ao escore z -2 da distribuição de referência revelam atraso do crescimento linear da criança e indicam, portanto, desnutrição de longa duração, seja decorrente de deficiência de energia, seja decorrente de deficiência de macro ou micronutrientes. Valores do IMC para idade inferiores ao escore z -2 revelam acúmulo insuficiente de massa corporal ou, mais frequentemente, utilização dos tecidos corporais para produção de energia, indicando, nesta situação, quadros de desnutrição. Valores do IMC para idade superiores ao escore $z+2$ indicam acúmulo de tecido adiposo e são usualmente utilizados para diagnosticar a condição de excesso de peso. Valores do índice altura para idade superiores ao escore $z+2$ são raros em todas as populações e não estão usualmente associados a distúrbios nutricionais. ${ }^{8}$

O protocolo foi revisado e aprovado pelo Comitê de Ética e Pesquisa do HUPEUERJ, cadastrado pelo registro CEP/HUPE - 2830/2010. O genitor (ou representante legalmente autorizado) que acompanhou a criança à consulta inicial foi previamente informado quanto à natureza e ao propósito do estudo, bem como esclarecido previamente à realização de qualquer procedimento relacionado ao protocolo que possa ser realizado, assinando um termo de consentimento livre e esclarecido.

\section{Resultado}


Uso de fórmula

inadequada no

primeiro ano de

vida

Orientação para AM

No pré-natal

$85,7 \%(66)$

$\mathrm{Na}$ maternidade

$96,4 \%(74)$

$\mathrm{AM}^{1}$

$$
\text { A qualquer momento }
$$

$98,8 \%(76)$

$8,9+3,92$

Com um ano de

$51,9 \%(40)$

duração

$\mathrm{AM}^{1}$ exclusivo

Idade média de

introdução

Fórmula láctea

Com duração de

$58,4 \%(45)$

quatro meses

Com duração de seis

$23,3 \%(18)$

meses

Água, suco ou chá

$4,7+1,7$

$4,6+3,3$

Alimentação

$5,3+1,2$

complementar

1-aleitamento materno; 2-média de tempo de AM; 3-média de tempo de AM exclusivo. 
Tabela 1- Caracterização do aleitamento materno ao longo do primeiro ano de vida incluindo frequências de orientação no pré-natal e na maternidade, de aleitamento materno (exclusivo ou não) a qualquer momento e com um ano de idade, e média de idade de aleitamento materno (exclusivo ou não) e de introdução de alimentação complementar.

A tabela 1 mostra os resultados encontrados, caracterizando o aleitamento materno ao longo do primeiro ano de vida em relação à frequência de orientação para aleitamento materno no pré-natal e na maternidade, e frequência de aleitamento materno, e aleitamento materno exclusivo, além de média de tempo de aleitamento materno e aleitamento materno exclusivo, bem como idade média de introdução dos alimentos complementares. A classificação do estado nutricional com um, dois, três, quatro e cinco anos de idade completos podem ser vistas nas figuras 1, 2, 3, 4 e 5, respectivamente.

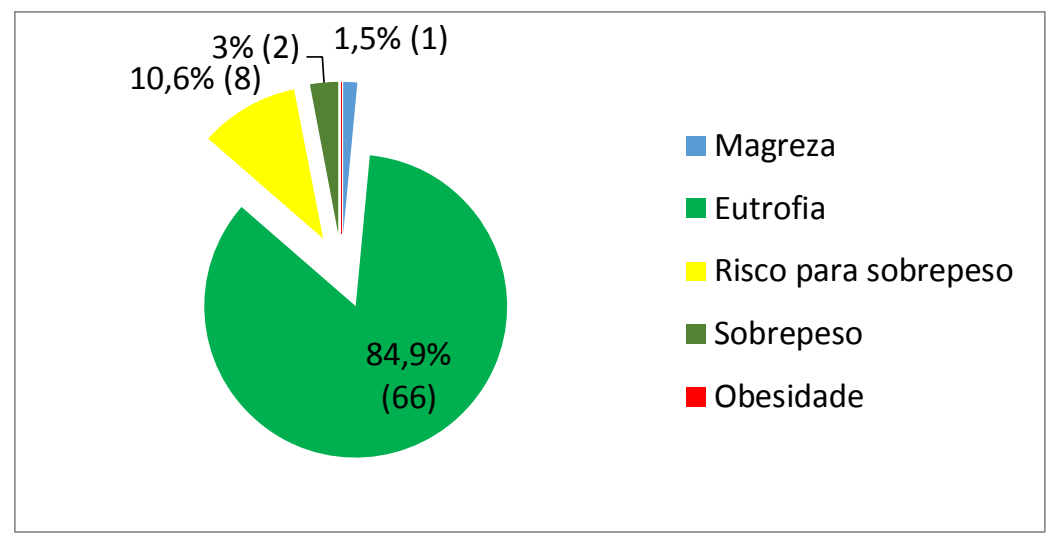

Figura 1 - Estado nutricional das crianças ao completar um ano de idade. 


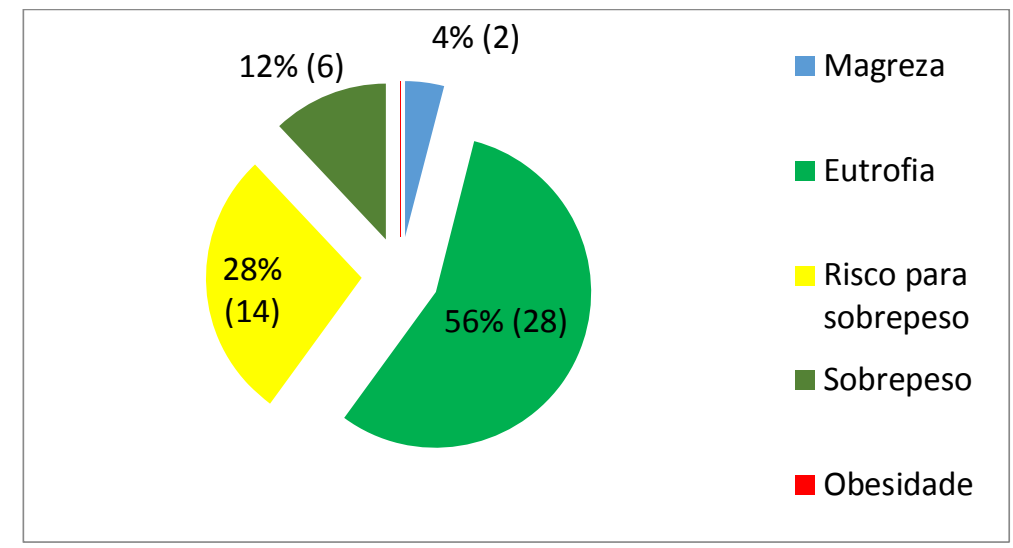

Figura 2 - Estado nutricional das crianças ao completar dois anos de idade.

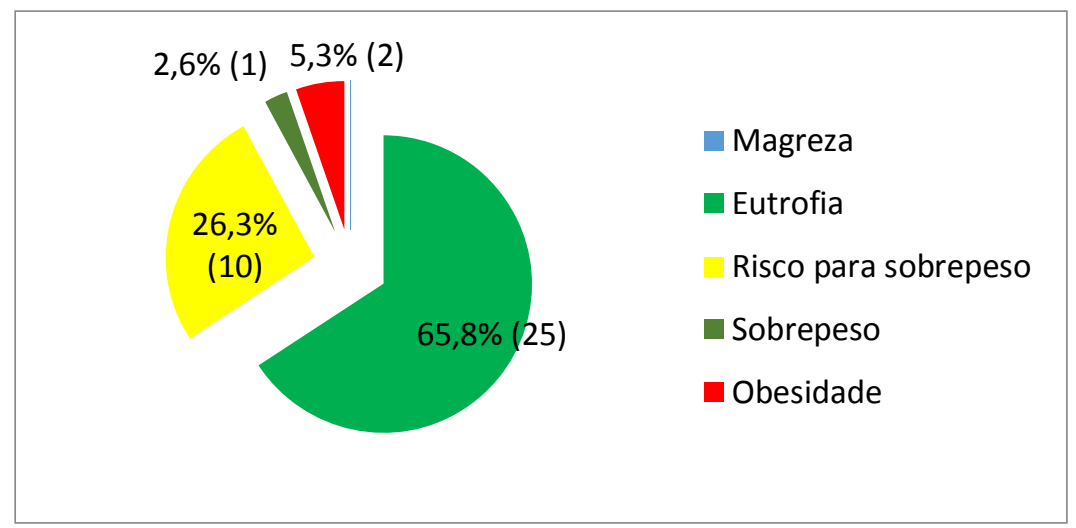

Figura 3 - Estado nutricional das crianças ao completar três anos de idade.

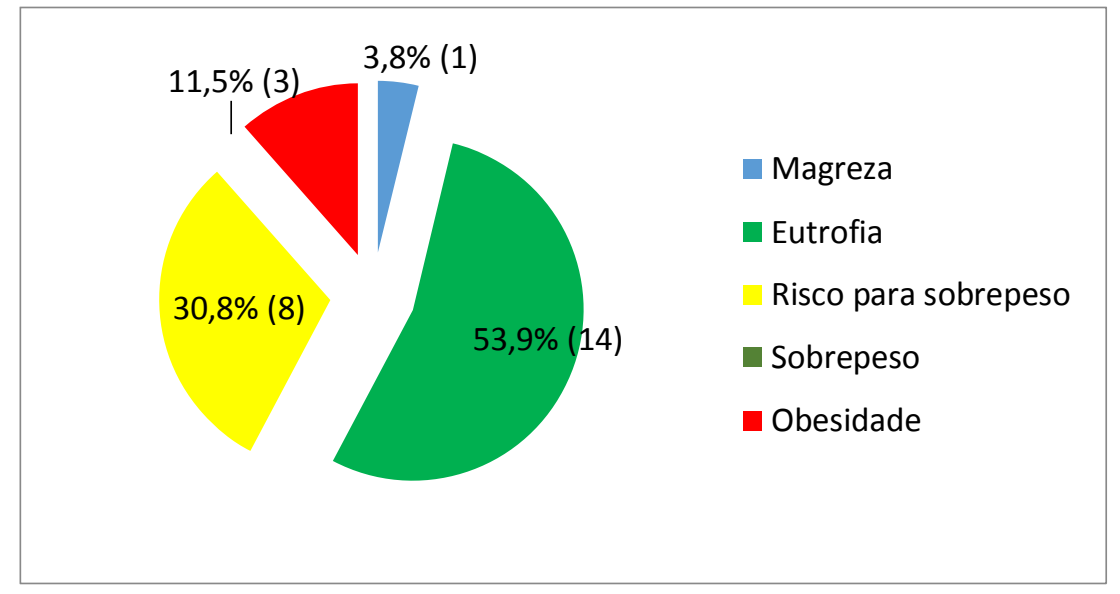

Figura 4 - Estado nutricional das crianças ao completar quatro anos de idade. 


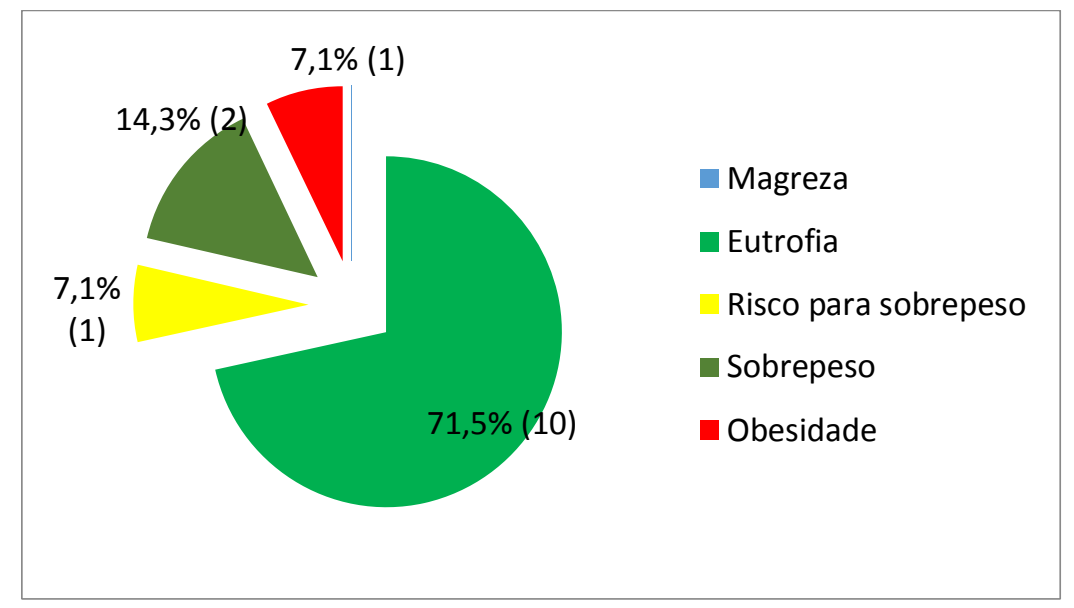

Figura 5 - Estado nutricional das crianças ao completar cinco anos de idade.

Pode-se observar que a maioria das mães foi orientada sobre a amamentação durante o pré-natal e/ou na maternidade, resultando na grande prevalência de aleitamento materno exclusivo a qualquer tempo (89,7\%), e de aleitamento materno (de qualquer natureza) a qualquer tempo $(98,8 \%)$, o que pode justificar a predominância de crianças não obesas em nosso projeto.

Quando se compara estes dados de aleitamento materno aos obtidos por Venancio et $\mathrm{al}^{9}$, em municípios do estado de São Paulo, percebe-se ainda que a frequência de aleitamento materno se mostra superior em nosso projeto, visto que em tal estudo foram encontradas frequências maiores que 20\% de aleitamento materno exclusivo maiores apenas em 27 municípios avaliados e de aleitamento materno, em 72 de um total de 84 municípios. Tais resultados, como o próprio autor afirma, podem estar relacionados à pequena quantidade de hospitais com o título de Hospital Amigo da Criança na região estudada, o que provavelmente diminui muito as orientações sobre aleitamento às gestantes e puérperas. A este respeito, estimou-se que as crianças que não nasceram em um Hospital Amigo da Criança, teriam uma chance 2,2 vezes maior de receber alimentação complementar, bem como uma chance 1,5 vezes maior de não estar em aleitamento materno exclusivo antes dos quatro meses de vida. ${ }^{9}$

A pesquisa revelou que a maioria das crianças acompanhadas no Projeto AMAR 
até o quinto ano de vida, como apresentado no Gráfico 1, foi classificada como eutrófica respectivamente do $1^{\circ}$ ao $5^{\circ}$ ano: $84,9 \%, 56 \%, 65,8 \%, 53,9 \%$ e $71,5 \%$ - e, até o segundo ano, com ausência de obesidade.

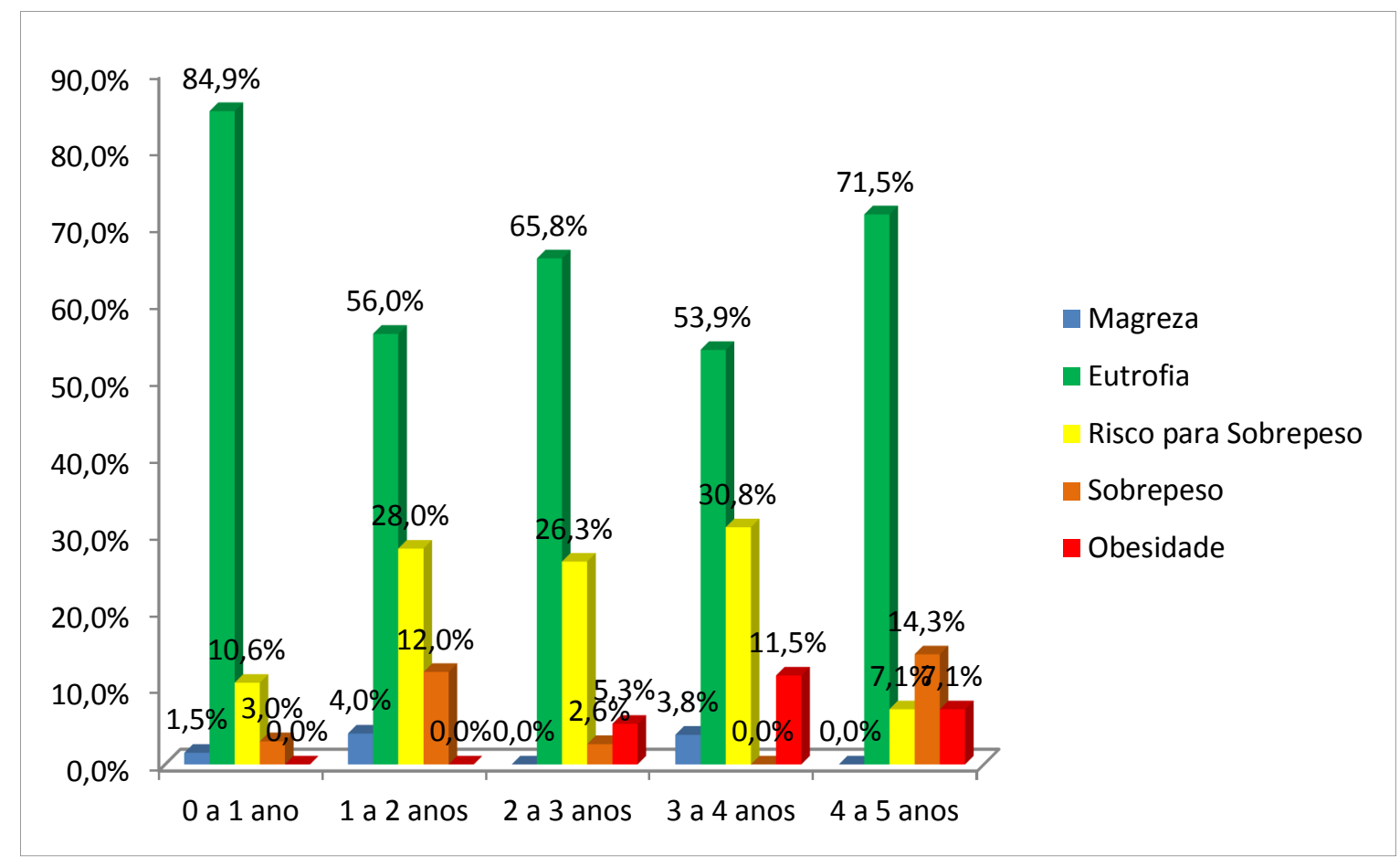

Gráfico 1 - Estado nutricional das crianças ao completar um, dois, três, quatro e cinco anos de idade. Projeto AMAR, DPED HUPE/UERJ - Rio de Janeiro, 2006-2014

No que se refere ao índice altura por idade, segundo a classificação da OMS8, todas as crianças acompanhadas pelo Projeto AMAR foram classificadas com estatura adequada para a idade. Não foram encontradas crianças com risco para baixa estatura ou baixa estatura para a idade.

\section{Discussão}

O AMAR proporciona uma abordagem integral e orientação multidisciplinar em diversas áreas de saúde com estudantes e profissionais médicos, nutricionistas, enfermeiros, fonoaudiólogos, assistentes sociais e psicólogos. O incentivo ao aleitamento 
materno se faz indispensável para a nutrição e desenvolvimento adequados nos primeiros anos e deve ser reforçado, especialmente no primeiro ano e exclusivamente durante os seis primeiros meses. Os resultados encontrados em relação à frequência de orientação quanto ao aleitamento materno no pré-natal e na maternidade e quanto à frequência de aleitamento materno (exclusivo ou não), ao tempo médio de aleitamento materno e de aleitamento materno exclusivo, e idade média de introdução dos alimentos complementares, demonstra a relevância da orientação dada pela equipe do projeto, quando se compara com a literatura. ${ }^{4}$

Na Pesquisa de Orçamentos Familiares (POF) 2008/2009 nota-se que a curva de peso das crianças brasileiras vem se afastando dos padrões ditados pelas curvas da OMS, independentemente da idade e do sexo, apontando para índices de excesso de peso. Tal observação difere do encontrado neste estudo, tendo em vista a maioria das crianças ser eutrófica em todas as faixas etárias analisadas, como pode ser observado nas figuras 1, 2, 3, 4 e 5, o que provavelmente está relacionado com o acompanhamento multidisciplinar realizado no projeto AMAR, incluindo profissionais da nutrição, com ênfase na orientação nutricional na infância. ${ }^{10}$

Segundo Cocetti et $\mathrm{al}^{11}$ as altas taxas de excesso de peso encontradas no Brasil são atribuídas principalmente ao abandono precoce do aleitamento materno, aumento da renda e maior disponibilidade de alimentos industrializados, bem como à veiculação de propaganda de alimentos de alta densidade calórica e baixo valor nutricional. Do mesmo modo, famílias cuja renda per capita foi maior que um salário mínimo apresentaram 2,5 vezes mais chance de excesso de peso. Na região sudeste, no entanto, a partir de 1989, foi observado queda de $0,5 \%$ ao ano na prevalência de excesso de peso. Tal diminuição seria explicada pelo incentivo ao aleitamento materno, maiores taxas de escolaridade materna e acesso à informação e educação sobre saúde, nutrição e dietética infantil.

Por sua vez, a POF de 2008/2009 demonstra uma forte tendência de diminuição da prevalência de déficit de altura com o aumento da renda (de 8,2\% no estrato de menor renda - menor ou igual a $1 / 4$ salário mínimo - para 3,1\% no estrato de maior 
renda - maior que cinco salários mínimos per capita), denotando a forte influência que a renda familiar ainda exerce sobre o risco da desnutrição infantil no Brasil. A prevalência de déficit de altura em menores de 5 anos de idade foi semelhante em meninos e meninas: 6,3\% e 5,7\%, respectivamente, sendo a prevalência de déficit de altura máxima no primeiro ano de vida $(8,4 \%$ e $9,4 \%$, respectivamente em meninos e meninas), diminuindo para cerca de $7,0 \%$ no segundo ano e variado entre $4,0 \%$ a $6,0 \%$ na faixa etária de 2 a 4 anos. $^{10}$

Com relação à desnutrição, dados dessa mesma pesquisa mostraram que a desnutrição e o baixo peso vêm declinando em comparação com a década de 80 . Em tal estudo, para faixa etária menor que 5 anos, foi utilizada a medida de déficit ponderoestatural como forma de aferição da prevalência de desnutrição e baixo peso, sendo encontrada para a região sudeste, uma prevalência de $6,1 \%$ de déficit de altura, caracterizado por um distanciamento maior ou igual a z-score -2 na curva de peso por idade da OMS.10 No Projeto AMAR, por sua vez, foi utilizado o cálculo de IMC para a classificação nutricional das crianças assistidas. A partir disso, foram encontradas, em todas as faixas etárias avaliadas, frequências de magreza abaixo das encontradas na POF para a mesma região do país na qual o projeto em questão está inserido, como se pode avaliar nas figuras 1, 2, 3, 4 e 5, também refletindo o trabalho multidisciplinar realizado no mesmo. No entanto, deve-se destacar que o grupo da POF utilizou parâmetros distintos desta pesquisa para aferir o estado nutricional das crianças, no que diz respeito ao déficit ponderal.

Apesar das orientações da equipe multidisciplinar sobre aleitamento materno e alimentação ao longo da infância, a presença de outros fatores que podem vir a influenciar negativamente os benefícios desta abordagem - como o trabalho materno, estrato social e preço alto de fórmulas lácteas adequadas, bem como fatores emocionais e falta de suporte familiar - podem justificar a frequência ainda significativa de excesso de peso, seja risco para sobrepeso, sobrepeso ou obesidade, nas crianças estudadas. Esses fatores condicionam à introdução da alimentação complementar em período inadequado 
ou mesmo ao uso de alimentação inadequada hipercalórica, como o leite de vaca. ${ }^{12,13}$ Tais informações podem ser avaliadas na tabela 1, onde: a média de introdução da alimentação complementar ficou em torno dos 5,3 meses; a média de introdução de água, sucos e chás em torno dos 4,7 meses; e a frequência de inadequação no uso de fórmulas lácteas foi de $46 \%$.

Em especial, ainda dentre os fatores de influência sobre o aleitamento materno, destaca-se a Consolidação das Leis do Trabalho (CLT) e a Constituição Brasileira que garantem à mãe 120 dias de licença-maternidade sem prejuízo do emprego ou salário. Apesar de favorecer o aleitamento materno inicialmente, este regime acaba por limitar o aleitamento materno exclusivo aos primeiros quatro meses de vida do bebê, e a inserção de alimentação complementar precocemente. No serviço público, em contrapartida, as mães têm garantido o direito de estender por seis meses a licença maternidade, desde 2008. Nos serviços particulares, a partir de 2010, existe um incentivo a essa prorrogação, de forma que as empresas que o concedem têm ressarcimento integral em impostos federais. Tais medidas proporcionam um início de vida potencialmente mais saudável para essas crianças. ${ }^{14}$

Por outro lado, sabemos que grande parte da população que compõe a clientela do HUPE-UERJ não está inserida formalmente no mercado de trabalho, não possuindo os direitos trabalhistas descritos.

Analisando os fatores que podem influenciar o estado nutricional nos primeiros cinco anos de vida, destaca-se o aleitamento materno. Segundo Balaban e Silva15, a obesidade deve ser encarada como uma doença multifatorial, que envolve fatores genéticos e ambientais, o que incluiria as primeiras experiências nutricionais de um indivíduo. Dessa forma, o aleitamento materno tem influência no desenvolvimento de tal agravo, funcionando como um fator protetor. A este respeito, Simon et $\mathrm{al}^{16}$, não temem em afirmar tal associação a partir dos dados obtidos estudando pré-escolares em São Paulo. 
Especificamente no projeto AMAR, como exposto na tabela 1, pode-se observar que a maioria das mães foi orientada sobre a amamentação durante o pré-natal e/ou na maternidade, resultando na grande prevalência de aleitamento materno exclusivo a qualquer tempo $(89,7 \%)$, e de aleitamento materno (de qualquer natureza) a qualquer tempo $(98,8 \%)$, o que pode justificar a predominância de crianças não obesas em nosso projeto.

Estudos de coorte longitudinais têm associado o ganho de peso excessivo e obesidade infantil ao risco de mortalidade geral na idade adulta, principalmente ao de doença arterial coronária. Um estudo relatou dados de acompanhamento de longo prazo em 31 adolescentes com excesso de peso com síndrome metabólica em comparação com um grupo controle, sem síndrome metabólica na infância. Após vinte e cinco anos do início do estudo, $19,4 \%$ do grupo de síndrome metabólica pediátrica tinha experimentado um evento cardiovascular significativo em comparação com apenas 1,5\% do grupo controle, o que mostra a importância da atenção nutricional durante o acompanhamento do crescimento e desenvolvimento no lactente e no pré-escolar. ${ }^{17}$

\section{Conclusão}

O acompanhamento do ganho pondero-estatural de nossa clientela, quando comparado à literatura, mostrou frequências maiores de crianças eutróficas, e principalmente, uma frequência de obesidade que se distancia da literatura brasileira, apesar de esta prevalência ainda ser significativa. Tais achados, provavelmente se relacionam ao alto índice de aleitamento materno, ao fato das crianças estudadas serem originárias de um Hospital Amigo da Criança, e por fim, às orientações da equipe multidisciplinar. 


\section{Contribuição dos autores}

MANSUR, Fabiana Migliaccio - Trabalho de campo, compilação de dados, cálculos estatísticos, digitação e pesquisa em planilha do Excel, discussão dos resultados, redação e revisão do artigo.

MADEIRA, Isabel Rey - Planejamento da pesquisa, trabalho de campo, compilação de dados, cálculos estatísticos, discussão dos resultados, orientação e supervisão dos alunos, redação e revisão do artigo.

NARCISO, Lara Pimenta - Trabalho de campo, discussão dos resultados, redação e revisão do artigo.

COSTA, Ana Paula Córdova da - Trabalho de campo, orientação e supervisão em Fonoaudiologia.

MACHADO, Antônia da Conceição Cylindro - Trabalho de campo, orientação e supervisão em Enfermagem.

RIBAS, Simone Augusta - Trabalho de campo, orientação e supervisão em Nutrição.

MENESES, Celise Regina Alves da Motta - Trabalho de campo, orientação e supervisão dos alunos.

DARRIBA, Vinicius Anciães - Trabalho de campo, orientação e supervisão em Psicologia.

CARVALHO, Dayse Silva de - Trabalho de campo, orientação e supervisão em Assistência Social.

\section{Referências}

1. World Health Organization. Division of Health Promotion and Protection. Food and Nutrition Program. Guiding Principles for Complementary Feeding of the Breastfed Child. Washington D.C.:2001. 
2. BRASIL. Ministério da Saúde. Caderno de Atenção Básica nº 11. Saúde da Criança: Nutrição Infantil. Aleitamento Materno e Alimentação Complementar. Brasília: 2009.

3. FIGUEREDO, Sonia Fontes; MATTAR, Maria José Guardiã; ABRAO, Ana Cristina Freitas de Vilhena. Iniciativa Hospital Amigo da Criança: uma política de promoção, proteção e apoio ao aleitamento materno. Acta paul. enferm., São Paulo, v. 25, n. 3, p. 459-463, 2012. Disponível em: http://www.scielo.br/scielo.php?script=sci_arttext\&pid=S0103. 21002012000300022\&lng=en\&nrm=iso. Acesso em: 12/02/2016.

4. MADEIRA, Isabel $\mathrm{R}$ etal.Projeto AMAR - Assistência Multidisciplinar em Pediatria - Uma Experiência em Puericultura Multidisciplinar. In: Congresso Brasileiro de Ensino e Pesquisa em Saúde da Criança e do Adolescente, 2008, Rio de Janeiro. Revista de Pediatria SOPERJ 2008; VIX: 127.

5. Reaven, GM. Role of insulin resistance in human disease.Diabetes.1988; 37:1595607. Disponível em: http://www.ncbi.nlm.nih.gov/pubmed/3056758. Acesso em: 10/03/2016.

6. Zimmet $\mathrm{P}$, et al. The metabolic syndrome in children and adolescents. Lancet. 2007; 369:2059-61. Disponível em: http://www.ncbi.nlm.nih.gov/pubmed/17586288. Acesso em: 10/03/2016.

7. BRASIL. Ministério da Saúde. Protocolos do Sistema de Vigilância Alimentar e Nutricional- SISVAN na assistência à saúde. Brasília: Ministério da saúde, 2008.

8. World Health Organization. The WHO Child Growth Standards. Disponível em: www.who.int/childgrowth. Acesso em: 25/02/2016.

9. VENANCIO, Sonia Isoyama et al. Freqüência e determinantes do aleitamento materno em municípios do Estado de São Paulo. Rev. Saúde Pública, São Paulo, v. 36, n. 3, p. 313-318, June 2002. Disponível em:www.scielo.br/scielo.php?script=sci_arttext\& $\mathbf{p i d}=\mathbf{S} 0034$ 89102002000300009\&lng=en\&nrm=iso. Acesso em: 14/03/2016. 
10. Pesquisa de Orçamentos familiares 2008-2009. Antropometria e estado nutricional de crianças, adolescentes e adultos no Brasil. Ministério da saúde e Ministério do Planejamento, Orçamento e Gestão, IBGE. Disponível em: http://www.ibge.gov.br/home/estatistica/populacao/condicaodevida/pof/2008_ 2009_encaa/pof_20082009_encaa.pdf. Acesso em: 07/03/2016.

11. COCETTI, Monize et al. Prevalência e fatores associados ao excesso de peso em crianças brasileiras menores de 2 anos. J. Pediatr. (Rio J.), Porto Alegre, v. 88, n. 6, p. 503-508, 2012. Dec. Disponível em: http://www.scielo.br/scielo.php?script=sci_arttext\&pid=S002175572012000600010\&lng=en\&nrm=iso. Acesso em: 06/03/2016.

12. ARAUjO, Maria de Fátima Moura de et al. Custo e economia da prática do aleitamento materno para a família. Rev. Bras. Saúde Mater. Infant., Recife, v. 4, n. 2, p. 135-141, Junho 2004 . Disponível em: http://www.scielo.br/scielo.php?script=sci_arttext\&pid=S1519$38292004000200003 \& \operatorname{lng}=e n \& n r m=$ isso. Acesso em 26 Dez 2016.

13. DIEHL, Julia Polgati; ANTON, Márcia Camaratta. Fatores emocionais associados ao aleitamento materno exclusivo e sua interrupção precoce: um estudo qualitativo. Aletheia, Canoas, n. 34, p. 47-60, abr. 2011. Disponível em http://pepsic.bvsalud.org/scielo.php?script=sci_arttext\&pid=S1413. 03942011000100005\&lng=pt\&nrm=iso. Acesso em 26 dez. 2016.

14. Santiago et al. Direitos da Mulher Trabalhadora: na Gravidez, no Pós凹Parto e Durante o aleitamento Materno. Sociedade Brasileira de Pediatria, 2012. Disponível em: www.sbp.com.br/src/uploads/2012/12/Direitos-da-MulherTrabalhadora-na-Gravidez-no-Ps-Parto-e-Durante-o-Aleitamento-Materno.pdf. Acesso em: 10/03/2016.

15. BALABAN, Geni; SILVA, Giselia A. P. Efeito protetor do aleitamento materno contra a obesidade infantil. J. Pediatr. (Rio J.), Porto Alegre, v. 80, n. 1, p. 716 Feb. 2004.

Disponível em: 
www.scielo.br/scielo.php?script=sci_arttext\&pid=S0021-

75572004000100004\&lng=en\&nrm=iso. Acesso em: 14/03/2016.

16. SIMON, Viviane Gabriela Nascimento; SOUZA, José Maria Pacheco de; SOUZA, Sonia Buongermino de. Aleitamento materno, alimentação complementar, sobrepeso e obesidade em pré-escolares. Rev. Saúde Pública, São Paulo , v. 43, n. 1, p. 60-69, 2009. Feb. Disponível em: www.scielo.br/scielo.php?script=sci_arttext\&pid=S0034$89102009000100008 \& \operatorname{lng}=e n \& n r m=$ iso. Acesso em 14/03/2016.

17. Lemos et al. Obesidade e síndrome metabólica em adolescentes: implicações futuras. Faculdade de Medicina da Universidade de Coimbra. Coimbra, 2014. Disponível em: https://estudogeral.sib.uc.pt/handle/10316/27989. Acesso em: $22 / 04 / 2016$. 